\title{
LA CULTURA IN LOMBARDIA NELL'ETÀ TERESIANA
}

\author{
Nota del s.c. GIANMARCO GASPARI (*)
}

(Adunanza dell'8 giugno 2017)

SUNTO. - La prima metà del Settecento si configura come una fase preparatoria, alla cui conclusione si collocano due fatti che acquisteranno presto un notevole valore simbolico, la morte di Muratori e la pace di Aquisgrana. A Milano, il lascito di Muratori è presente soprattutto negli studi storici, ma anche nel progetto di una sempre più forte interazione tra cultura e società. La nuova generazione degli uomini del «Caffè» aggiunge alla lezione di Muratori la miscela esplosiva delle novità d'Oltralpe, a partire dalla rivoluzionaria gerarchia dei saperi "pratici" di cui si era fatta veicolo l'Encyclopédie di Diderot e d'Alembert. «Il Caffè», Dei delitti e delle pene, le Meditazioni sulla felicità e le Osservazioni sulla tortura, ma anche la poesia civile di Parini, consegnano al secolo successivo una riflessione articolata sulla giustizia, sulla convivenza sociale e sul perfezionamento dell'uomo, che metteranno radici profonde nella coscienza comune, e ridurranno così la distanza che fino a tutto il Seicento separava l'Italia dall'Europa della scienza.

ABSTRACT. - The first half of the $18^{\text {th }}$ century, in a way, is a period of preparation, ending with two facts that will soon acquire a considerable symbolic significance: the death of Ludovico Antonio Muratori and the Treaty of Aix-la-Chapelle. In Milan, Muratori's legacy mainly lies in the historical studies, but it can also be seen in the project of an increasingly strong interaction between culture and society. The new intellectual generation, gathering around the periodical "Il Caffè", adds to what Muratori had taught the explosive mixture of novelties from the transalpine countries, first of all, Diderot and d'Alembert's Encyclopédie, and the revolutionary place it gives to 'practice' within the hierarchy of knowledge. "Il Caffè", and works such as On Crimes and Punishments, Meditations on Happiness, and Observations on Torture, but even Parini's civil poetry,

(*) Università degli Studi dell'Insubria, Varese-Como, Italy.

E-mail: gianmarco.gaspari@uninsubria.it 
hand down to the following century a multifarious reflection on justice, social coexistence, and improvement of mankind, which will become deeply rooted in the collective consciousness, thus reducing the $17^{\text {th }}$-century gap between Italy and Europe regarding science.

Una necessaria premessa al mio intervento era stata affidata all'amico Carlo Capra, che avrebbe chiarito da par suo le modalità istituzionali attraverso le quali si realizzano in Lombardia la gestione amministrativa di Maria Teresa e i cambiamenti che il suo governo ebbe modo di definire rispetto al passato. Nella divisione che avevamo concordato, a me spettava entrare nel vivo della vicenda culturale di quell'età, in particolare dei fatti legati a Milano, con un perno del discorso fissato agli anni centrali di tale vicenda, cioè, grosso modo, il ventennio Cinquanta-Settanta. Al professor Capra non è però stato possibile intervenire, e sarà quindi inevitabile avvertire l'assenza del quadro storico di riferimento.

In tutti i casi, è ben noto che con la salita al potere di Maria Teresa, nel 1740, si avvia un processo in cui l'Europa, grazie non solo alla definizione di trattati di pace ma soprattutto all'accorta politica matrimoniale tessuta dall'imperatrice (Tu, felix Austria, nube), conoscerà un sistema di equilibri e di alleanze che si prolungherà fino alla chiusura di quello che per consuetudine siamo soliti chiamare l'Antico Regime. Si parla dunque di un'epoca decisiva anche per i rivolgimenti successivi, che compressero in passaggi rapidissimi i rovesciamenti rivoluzionari, le clamorose affermazioni dei nazionalismi e i tentativi di restaurazione.

Per l'Italia come per il resto d'Europa, non è tanto l'anno in cui Maria Teresa assume il potere come «imperatrice regina» a rappresentare il tornante decisivo, quanto quello della pace di Aquisgrana, pace che segna una cesura formidabile nel secolo, anche per la valenza simbolica che assumerà quel 1748. Il trattato di Aquisgrana prepara all'Europa quasi cinquant'anni di pace, qualcosa che mai aveva conosciuto nelle ère precedenti. Mezzo secolo di pace, fatto salvo l'intervallo tra 1757 e '63, la guerra cioè dei Sette anni: nella quale, è vero, ebbe parte una personalità come Pietro Verri, che ne parlò a lungo come della propria unica esperienza d'armi, ma che possiamo decisamente collocare sullo sfondo, non incidendo più che tanto su questa singolare consapevolezza. Un mezzo secolo di pace che si è fatto condizionante anche per il nostro modo di pensare, dato che alla pace siamo soliti far corrispondere l'accelerazione delle scoperte e delle conquiste tecniche, scientifiche, mediche, filosofiche e sociali, che ci hanno portato inevita- 
bilmente a considerare come empiricamente il progresso si leghi alla pace stessa (dubito poi che l'Europa dell'Ottocento abbia riflettuto abbastanza su questo tema, che forse meriterebbe qualche riflessione anche nel nostro presente).

Sta di fatto che per Milano e la Lombardia la svolta decisiva si colloca subito dopo Aquisgrana, alla metà esatta del secolo. Nel 1750 muore Ludovico Antonio Muratori: una personalità che per la Milano della prima metà del secolo aveva costituito un riferimento essenziale. L'attività di prefetto alla Biblioteca Ambrosiana, tra il 1695 e il 1700, si chiude con la pubblicazione della Vita di Carlo Maria Maggi, omaggio non tanto a una delle personalità più notevoli nell'epoca della crisi del barocco, quanto indicazione ai suoi successori di un percorso che allacciasse decisamente la letteratura alla vita civile (così nell'esordio del libretto: «Il tessere la vita di Carlo Maria Maggi è un beneficio dovuto alla memoria bensì d'un gran letterato, ma più alla saggia curiosità de' secoli avvenire [...]. È un potente stimolo a ben coltivar le lettere, il vedere da qualche amorevole penna conservate in vita ancor le azioni di chi con tal mezzo divenne glorioso...»). Ma Muratori lascia alla città anche il piano concreto di un grandioso progetto culturale, quello dell'edizione dei Rerum Italicarum Scriptores, che Filippo Argelati espone al conte Archinto nel 1718, ricavandone una convinta adesione: da cui la costituzione, per far fronte all'enormità dell'impresa, della Società Palatina (dalla sede della tipografia, al pianterreno del Palazzo Ducale), che dal 1721 metterà a disposizione dei collaboratori milanesi designati da Muratori le più ricche biblioteche della città. Il primo dei volumi progettati (da cinque destinati ad aumentare a venticinque) esce due anni dopo, stampato con piombi fusi su matrici olandesi, appositamente fatti arrivare da Venezia. Prima della conclusione della pubblicazione dei Rerum, nel 1751, era stata avviata anche la stampa delle Antiquitates Italicae Medi Aevi, l'edizione dell'Opera omnia di Carlo Sigonio e soprattutto, sottolineando la ricaduta municipale di quelle iniziative, della Bibliotheca Scriptorum Mediolanensium di Filippo Argelati. Lui, bolognese, l'oblato milanese Giuseppe Antonio Sassi (prefetto dell'Ambrosiana dal 1713) e il valtellinese Francesco Saverio Quadrio (bibliotecario a Milano del governatore Pallavicini) furono tra i più attivi nel confermare alla città quel ruolo nuovo di capitale editoriale, legato a doppio filo al mecenatismo della classe nobiliare, che è già la prefigurazione di un percorso che si definirà decisivo, per le vicende culturali di Milano, lungo i tre secoli successivi. 
Muratori, simbolo e artefice di questa fase preparatoria - perfetta, se la storiografia italiana avesse fatto proprie alcune delle categorie messe in circolo dagli studiosi d'Oltralpe, a dar corpo a un nostro «Preilluminismo» - è però anche l'autore di testi come la Pubblica felicità e il Trattato del governo della peste, che consegnano alla riflessione successiva, nel periodo che corre tra Beccaria e Manzoni, alcuni nuclei cruciali di un dibattito destinato a farsi sempre più ampio. E che l'anno dopo la morte di Muratori, nel 1751, a Parigi si inizi a pubblicare la grande Encyclopédie, è quasi il segnale di come questi richiami invitino a una convinta sintonia con il cambiamento che si sta così avviando nel resto d'Europa. Tutto ciò accade rapidamente, molto rapidamente. L'assunzione in carico dell'eredità di Muratori coincide di fatto con un brusco revirement, quello stesso che in quegli anni decisivi inizia a separare, come mai era accaduto prima, la generazione dei figli da quella dei padri. Ė quel che successe ai maggiori rappresentanti a Milano di quel cambiamento, Pietro Verri e Cesare Beccaria, che mostrarono come il serpe della «filosofia» potesse allora allignare anche in seno alla nobiltà più uggiosa e conservatrice.

Il nome scelto per il giornale che Pietro Verri aveva destinato a divulgare «cose varie, cose disparatissime, cose inedite, cose fatte da diversi autori, cose tutte dirette alla pubblica utilità», ossia «Il Caffè», è rimasto famoso, a collocare quell'incontro periodico con un pubblico sicuramente più fedele che numeroso sotto l'insegna goldoniana della piacevole conversazione, improvvisata fra i tavoli di una bottega dove si serve «caffè vero verissimo di Levante e profumato col legno d'aloe, che chiunque lo prova, quand'anche fosse l'uomo il più grave, l'uomo il più plombeo della terra, bisogna che per necessità si risvegli e almeno per una mezz'ora diventi uomo ragionevole».

«Un giornale», si legge nell'Encyclopédie (voce Journal), «dev'essere l'opera di una società di uomini colti, altrimenti vi si troveranno, in ogni campo del sapere, abbagli dei più grossolani». E il gruppetto di amici che dai primi anni Sessanta s'era riunito attorno a Pietro aveva saputo far tesoro del consiglio, non rifiutandosi nemmeno a quel che di più impegnativo gli veniva pure richiesto, di avere cioè a cuore «i progressi dello spirito umano», di «amare la verità» e di misurare tutto «con questi due criteri di giudizio». La fortuna del «Caffè», tra 1764 e'66, spiega meglio di ogni analisi come poté realizzarsi quel programma, dal quale erano discesi pressoché contemporaneamente, frutti altrettanto inattesi di un'eccezionale temperie, le Meditazioni sulla feli- 
cità di Pieno e, soprattutto, l'esile ma dirompente libretto di Beccaria, Dei delitti e delle pene.

Merita soffermarsi su cosa potesse voler dire a Milano, in quei primi anni Sessanta del Settecento, la scelta di accollarsi la battagliera missione di rinnovare dall'interno non soltanto il costume letterario e le trite consuetudini accademiche, ma il rapporto stesso dell'uomo di cultura con il tessuto sociale di cui è parte, nel quale rapporto «l'amore delle virtù, dell'onestà, dell' adempimento de' propri doveri» - parole trite allora non molto meno che oggi -, giocherebbero dunque un ruolo tutt'altro che secondario. Da qui l'accento posto sulle discipline pratiche, la definizione di specializzazioni inedite rispetto agli interessi sino ad allora coltivati dall'aristocrazia intellettuale della città, delle quali la rivista inizia a farsi veicolo.

Occorre, per intenderlo bene, abbassare lo sguardo fino alla cronaca spicciola, alla biografia. Il conflitto di Pietro Verri con il padre, austero magistrato e autorevole membro dell'aristocrazia senatoria, rappresenta senz'altro il conflitto di due generazioni assolutamente incomponibili. La lingua di cultura di Gabriele Verri è il latino: quella di Pietro il francese. Alla rigida cultura giuridica del primo si oppone la curiosità intellettuale del secondo, la sua passione per la filosofia morale, le scienze sperimentali e applicate, l'economia, la storia. La stessa proposta, girata da Pietro a Beccaria, di occuparsi di un tema giuridico in un modo affatto diverso da quello che un giurista della generazione precedente avrebbe potuto scegliere, comportava non solo (e non si tratta certo di questioni secondarie) un parere diverso sulla tortura e sulla pena di morte: comportava anche che Dei delitti e delle pene venisse, caso eccezionale per un testo giuridico del Settecento, scritto in italiano e non in latino: ché, certo, non saremmo allora qui a parlarne. Pietro pagò caro quello scontro: «l'Accademia dei Trasformati e mio padre mi avevano rovinato l'anima», lascerà scritto lapidariamente di quella fase della sua giovinezza. L'indicazione è preziosa anche per l'altro bersaglio, l'Accademia dei Trasformati, appunto, presso la quale Pietro trascorse qualche anno prima di decidere che il suo avvenire, meglio che a Milano, potesse affidarsi a una guerra, quella dei Sette Anni, che abbiamo già ricordato.

La guerra lo condusse a Vienna: e a Vienna, proprio sul crinale del decennio, nel 1759, ebbe modo di incontrare il capo del Dipartimento d'Italia e dei Paesi Bassi austriaci, il conte - poi principe - Wenzel Anton von Kaunitz-Rittberg: un personaggio colto ed eccentrico, di for- 
mazione intellettuale notevolmente avanzata per un austriaco di metà Settecento. Aveva studiato all'università protestante di Lipsia, conosceva le opere maggiori del preilluminismo inglese ed era stato nei primi anni Quaranta ambasciatore a Torino, e poi, dal 1750 al '53, a Parigi. Non meno notevole il suo cursus bonorum politico, entro il quale fa spicco l'abilità dimostrata nelle trattative per la pace di Aquisgrana, che valse a promuoverlo a personaggio indispensabile al sistema, una volta che ne fosse deciso, come in effetti accadde, l'allontanamento dei sostenitori del più rigido centralismo burocratico.

Di Kaunitz, è rimasta celebre la battuta, giudicata concordemente imperdonabile da parte italiana, per cui si disse in grado di governare gli affari di Lombardia «nel tempo che si metteva le scarpe», e che, per quanta poca fede si debba concedere agli aforismi dei grandi, andrà comunque commisurata alla strabiliante lunghezza delle sue toilettes, come ci vengono presentate anche dall'osservatorio delle lumières parigine, tra Marmontel e d'Alembert. «Il m'avoit pris en amitié», ricorda il primo della fastosa ospitalità dell'ambasciatore d'Austria:

J'allais assez souvent dîner chez lui, au Palais Bourbon, et il me parlait de Paris et de Versailles en homme qui les voyait bien. Cependant je dois avouer que ce qui me frappait le plus en lui était la délicatesse et la vanité d'une âme efféminée. Je le croyais plus occupé du soin de sa santé, de sa figure, et singulièrement de sa coiffure et de son teint, que des intérêts de sa Cour.

Dalle pieghe di questa cronaca minore balza evidente un fatto che più ci preme, oggi, dell'acconciatura e della salute del ministro, e che la voluta noncuranza del narratore sottolinea in tutta la sua portata negativa: perché è chiaro che la scarsa dedizione alla propria carica poco o nulla poteva interessare la cerchia dei philosophes, là dove il suo stesso rendiconto si fa invece vistoso segnale di una mancata intesa. Se ne ha conferma proprio nella testimonianza viennese di Pietro, quando, trentenne in cerca di gloria, ebbe dunque modo di incontrare per la prima volta il ministro per ringraziarlo del grado di capitano appena ottenuto. Trascorriamo pure sulla coreografia di quell'incontro, che conferma appunto che per udienze come quella Kaunitz non disponesse di più tempo che per levarsi da tavola o per fare ingresso a teatro; e così sulla stessa fisionomia del conte, altero, elegante ma affettato (tratti resi perfettamente anche nel ritratto a pastello di Liotard, del 1762), e sulla sua cultura e la padronanza delle lingue («sembra un francese o un italiano 
ogni volta che cambia linguaggio»); non però sulla nota che chiude la descrizione della visita: «Fui contento», vi registra Pietro, «ma non lasciò di farmi specie la confidenza di trattarmi col voi, avendo io anche la chiave di ciambellano». Quello, gli spiegò poi il suo accompagnatore, era di norma l'atteggiamento tenuto da Kaunitz con i milanesi.

Un conte del Sacro Romano Impero apostrofato col vocativo dei subalterni, si dirà, non è fatto che possa dare la misura di chi davvero fu il principe di Kaunitz: non certo meglio di quanto lo darebbe l'esame della sua politica liberistica o delle sue innovazioni in campo burocratico e sociale. Un dato, tuttavia, non andrà trascurato da chi insista a muovere dalle fonti per approfondire la storia di quest'età: ed è che il tratto di quella scostante alterigia, così come la distanza risolutamente segnata tra la sua statura intellettuale di austriaco di metà Settecento e la nuova cultura dell'Europa delle lumières - decisamente scelta e fatta propria dalla parte più avvertita, e per numero e peso tutt'altro che irrilevante, dell' intelligentsia lombarda -, di questa storia fanno precisamente parte: si tratterà di assegnare loro, naturalmente, una giusta misura in proporzione al resto. Ora, se il nostro osservatorio si conferma quello di parte milanese, vi assume un ruolo di prima importanza proprio la testimonianza di Pietro, disposto dal singolo incontro con Kaunitz a muovere, nel giro di poche pagine, a un più ampio confronto tra le due nazioni. Ed è pagina che va riportata per intero, in limine a ogni possibile dibattito sui rapporti tra lo stato vassallo e quello sovrano, anche quando si vada a dibattere di un'età felice come quella seguita, per l'Italia, alla firma della pace di Aquisgrana: «Generalmente», scrive dunque Verri dell'atmosfera respirata a Vienna sul principio dell'estate di quel 1759,

Generalmente questi signori Austriaci ci guardano come provinciali, come li Inglesi guarderebbero li Americani loro sudditi. Un galantuomo di merito e modesto può guardarsi come perduto: non s'accorgeranno mai che un uomo abbia cognizioni e spirito s'egli medesimo sfrontatamente non glielo ripete, e non conviene ributtarsi per freddezza o sgarbo, ma instare, proseguire, farsi avanti e parlar alto, fermo e decisivo. lo vedo uomini ben da poco, che con questa scuola vanno festeggiati e ben accolti [...]. Ho osservato che in questa città capitale non vi son forestieri di sorte alcuna, se non quelli che per officio o speranza vi soffrono il soggiorno [...]. Si credono di buona fede questi Austriaci superiori al restante d'Europa, se ne eccettui Parigi e Londra, che hanno i loro partigiani anche qui. Quante siano poi nelle biblioteche le opere d'ingegno prodotte in questo clima e da quali nazionali, non saprei, non conosco un celebre pittore, non un architetto illustre che sia da annoverarsi fra li 
Austriaci, e nemmeno saprei se in tutta la monarchia abbia la Casa d'Austria una città che sia paragonabile a Milano per ogni riguardo [...]. Se non sapete il tedesco, vostro danno, essi non hanno l'attenzione che abbiamo noi in Italia di usare del francese quando vi sia un forastiere che non sappia la nostra lingua; non s'incomodano punto perciò: vi invitano a pranzo, le tavole sono assai ben servite, ma talvolta vi è un silenzio stupido che vi annoia mortalmente nel tempo che pure altrove è destinato alla giocondità ed alla amicizia.

Difficile per Pietro sottrarsi poi, e sia pure in un futuro che vide di molto aumentata la stima di Vienna per Milano e i suoi abitanti, alla memoria tenace di considerazioni come questa, che ben altro chiamavano in causa che le mal ripagate ambizioni di un rampollo di buona famiglia. Con ciò, va detto che restando vero quel che la storiografia recente non s'è stancata di affermare, e cioè il carattere eccezionale dell'intesa riformatrice tra la Dominante e lo Stato di Milano, restano tuttavia vere e necessarie le distinzioni da compiere, che consentiranno di non stupirci del prosieguo degli eventi, del venire a sapere ad esempio con quale fermezza Kaunitz continuasse a ritenere «meri sudditi»e, come tali non formanti «corpo di Stato» i Milanesi, per ricercare contemporaneamente (e talvolta ottenere) l'intesa con lo stesso Verri, che di quel concetto di sudditanza fu tra i più strenui oppugnatori. La razionalizzazione dell'apparato amministrativo che era nelle mire di Kaunitz, di fatto, non giunse conseguentemente mai a tradurre in concreto quelle che davvero furono le ambizioni del pensiero illuministico: e questo nonostante il favore concesso a scienziati e scrittori che ne furono tra i massimi portavoce in Europa (e che, proprio per l'ampia rispondenza della loro opera, sollevarono addirittura l'impressione, parallelamente perpetuata dallo sciovinismo francese e dal moderatismo italiano, che i «lumi» d'Italia non fossero più che emanazione e imitazione di quelli d'Oltralpe), da Beccaria a Paolo Frisi, posti al centro dei delicatissimi apparati della censura e dell'istruzione superiore: per Beccaria, si ricordi anche come Kaunitz, offrendogli e anzi istituendo per lui una delle prime cattedre europee di materie economiche, ne impedì la fuga in Russia, dove l'aveva chiamato Caterina II perché vi attendesse alla riforma del Codice. Che era quanto poteva bastare a un letterato veneto, ma con buoni contatti in Lombardia, per sottolineare la singolarità di un'epoca nella quale vigeva «il costume che i principi chiamassero alla cattedra i letterati, non i letterati domandassero la cattedra ai principi». 
La ricerca di nuove dimensioni nell'esercizio del potere, che è tra i punti cruciali del pensiero politico dell'illuminismo, si dichiara nello stesso concetto cui l'Austria diede vita proprio con le riforme avviate negli anni Cinquanta anche negli stati ereditari, quello cioè di dispotismo illuminato. E percorrere, come i fatti li obbligarono, la via austria$\mathrm{ca}$, per gli intellettuali milanesi comportò sì fedeltà, non comunque rinuncia alla propria orgogliosa autonomia di pensiero, anche se una significativa convergenza va registrata proprio sul tema del dispotismo, pienamente accettato come ottima delle soluzioni all'atto dello stabilimento delle riforme (fu la posizione di Pietro Verri e dell'allievo suo Giuseppe Gorani): prova esemplare, questa, di un pragmatismo che si definì con risolutezza nell'avviamento delle élites intellettuali alle carriere amministrative, e che segna evidentemente il punto estremo del distacco dalla teoria dei philosophes: «Ve li immaginate Diderot e Rousseau alti funzionari?», suona ancora efficace la domanda con cui Franco Venturi, nel 1954, prefigurava il nuovo corso degli studi settecenteschi in Italia: «Beccaria e Verri lo furono».

Dopo la dismissione del «Caffè», infatti, e come per tempo aveva fatto Pietro, anche Cesare Beccaria, per un paio d'anni impegnato nell'insegnamento della Pubblica economia presso le Scuole Palatine (la scuola d'istruzione superiore della capitale, cui non toccava però il titolo di Università, prerogativa riservata alla sola Pavia), era entrato nei ranghi dell'amministrazione austriaca come alto funzionario del Magistrato Camerale. Un cursus honorum singolare, quello che li condusse dalla combattiva e "antagonistica" esperienza del «Caffè» al ruolo di civil servant, ma che il quadro complessivo, credo, potrà ampiamente giustificare, nel senso di una fattiva intesa - per più versi eccezionale nel percorso storico del nostro paese - tra potere centrale e responsabilità e ambizioni della nuova classe dirigente.

È quanto contribuisce a spiegare il caso, più esemplare che eccezionale in quel preciso contesto storico-culturale, della nascita della Biblioteca Braidense. Il fondo originario di quella formidabile raccolta libraria apparteneva al conte Carlo Pertusati, dal 1733 presidente del Senato di Milano. Morto nel '55, il conte aveva fatto in tempo a veder celebrata la sua biblioteca, ricca di oltre ventiquattromila volumi, da estimatori occasionali come dai primi agguerriti esploratori di ogni più impervio documento dell'italico sapere, a partire appunto dalla scuola di Muratori. «Nulli ex privatis secundam», quella biblioteca non meritava la dispersione e l'oblio cui la più tiepida passione del conte Luca, 
il figlio del raccoglitore, l'avrebbe presto o tardi destinata. Ed ecco farsi avanti, ad ammonire la Congregazione dello Stato circa l'entità della possibile perdita, proponendone al tempo stesso il rimedio, il ministro plenipotenziario, Carlo di Firmian, un tirolese che segnò con la sua decisiva presenza buona parte della vita sociale e culturale della Lombardia settecentesca.

Aveva studiato a Ethal, a Innsbruck e a Salisburgo. Suo fratello era principe vescovo di Lavant, in Carinzia; lui, signore di Cronmetz, Meggel e Leopoldscron: nomi e luoghi che dovevano mettere i brividi a ogni buon milanese di quell'età, per natura sospettoso di tutto quel che provenisse dal Nord e tanto più dei tedeschi, ai quali i tirolesi erano sbrigativamente assimilati. Ma Firmian aveva visitato Parigi e l'Olanda, Firenze e Roma. Era stato ambasciatore dell'Impero a Napoli. In qualche modo, aiutata magari dall'associazione all'Accademia degli Etruschi di Cortona, la sua presenza in Italia parrebbe richiedere per sintonia, più che quella degli armigeri coi baffoni di sego, la compagnia di un Winckelmann, o almeno di un Lord Hamilton. Non è tuttavia dato sapere quanto covasse in lui la stessa "vocazione all'Italia " che fu croce e delizia dell'età sua, né certo l'aria di Milano, pur respirata dalle finestre dello splendido palazzo Melzi, poteva consentire a paragoni con l'omerica solarità meridionale, quella che davvero calamitava gli artisti e gli adepti del Grand Tour - che nemmeno baderanno più che tanto, fino almeno agli anni di Stendhal, a dar peso alla capitale del Ducato nel progetto dei loro itinerari.

Comunque fosse, l'appello di Firmian a Maria Teresa fu decisivo, almeno quanto l'arrivo a Milano del benedettino Andrea Mazza, incaricato di valutare la biblioteca in vista di un possibile acquisto da parte delle autorità parmensi. Doveva essere ancora in corso la trattativa, che rischiava di sottrarre a Milano quel prezioso patrimonio, quando nel giugno del '63 il delegato di Cremona decideva di soprassedere a ogni ulteriore indugio e si dichiarava disposto all'acquisto. Gli altri deputati della Congregazione dello Stato ne seguirono immediatamente l'esempio, nonostante le difficoltà finanziarie in cui versava l'amministrazione: ma si trattava di ingraziarsi l'imperatrice, con un dono eccezionale, quale si poteva offrire non più di una volta in un secolo, e dunque davvero degno di lei e dell'augusto figliolo.

Firmian venne incaricato di fungere da intermediario fra la Congregazione e il conte Luca, e il contratto poté così essere stipulato nel giro di poche settimane. La Congregazione dello Stato si impegnò 
al pagamento, in sei rate, di duecentoquarantamila lire, e alla redazione di un catalogo che illustrasse alla sovrana le caratteristiche della raccolta, particolarmente ricca nel settore storico, ecclesiastico e delle «lettere umane». Il secondo compito fu di gran lunga più gravoso del primo, così che spesso giunsero da Vienna richiami e sollecitazioni, le lunghe more del lavoro riuscendo mal accette al solerte apparato centrale. Nel frattempo è da credere che il destinatario del dono in ragione della sua collocazione milanese, l'arciduca Ferdinando, avesse mostrato in qualche modo ai suoi sudditi e alla stessa imperatrice che un tale tesoro rischiava, nelle sue mani, di rimanere quantomeno inoperoso.

Il quattordicesimo del figli di Maria Teresa d 'Austria e di Francesco Stefano di Lorena nutriva infatti ben altre ambizioni che quella del bibliofilo. Ci è noto almeno che gli piaceva la musica, se per esempio nell'inverno del 1771 gli poté sorridere l'idea di trattenere a Milano, al suo servizio, il giovane musicista dell'Ascanio in Alba, composto - su versi di Parini - per celebrare il suo matrimonio con Maria Beatrice d'Este. Nel finale dell'opera, colpiscono le parole che Venere indirizza, in forma di congedo, allo sposo:

\author{
De le mie leggi \\ tempra il soave freno: \\ ministra il giusto: il popol mio proteggi. \\ In avvenir due numi \\ abbia in vece d'un sol: te qui presente; \\ me, che lontana ancora \\ qua col pensier ritornerò sovente.
}

Colpiscono perché sembrano adombrare il monito che Maria Teresa rivolge al figlio a séguito della sua richiesta, dove è evidente che per un tale argomento non aveva avuto necessità di consultare chicchesia, mostrando anzi quanto scarsa fosse la considerazione in cui erano tenuti i desideri di Ferdinando, fosse pure il governatore del Ducato:

Mi chiedi se devi prendere al tuo servizio il giovane salisburghese. Non vedo quali ragioni tu possa avere d'assumere un compositore o altre persone inutili. Se proprio ti fa piacere non voglio impedirtelo: te lo dico soltanto perché tu non ti carichi di gente inutile. In ogni modo evita di conferir titoli a simili persone, come se fossero al tuo servizio. Poiché quando si mettono a girare il mondo come mendicanti discreditano il servizio. 
Se il tono tanto poco incoraggiante dell'imperatrice tolse a Milano l'occasione di ospitare più a lungo il giovane Mozart, può forse convergentemente spiegare perché, in quel giro di mesi, l'arciduca che godeva di una simile stima fu, malgré soi, la causa che ottenne alla città la futura biblioteca Braidense, una biblioteca finalmente destinata «ad uso comune di chi desidera di maggiormente coltivare il proprio ingegno e acquistare nuove cognizioni». Rimaneva, come ben chiariva il rescritto, il problema della scelta del «sito opportuno» dove collocarla: «per quanto sarà possibile vicino al centro della Città, di facile e comodo accesso».

Nel luglio del 1773, pochi mesi dopo che Giambattista Castiglione annunciò alle autorità la chiusura del catalogo delle opere incomplete della Biblioteca Pertusati, papa Clemente XIV emanava il breve Dominus ac Redemptor, con cui veniva dichiarato lo scioglimento della Compagnia di Gesù. Al di là dei clamorosi rivolgimenti che ne seguirono, la conseguenza immediata per la sorte dell'ex biblioteca Pertusati fu, di fatto, estremamente positiva: lo Stato acquisiva la proprietà del Collegio Braidense del Richini, che per gli spazi a disposizione, la razionalità dell'impianto e la collocazione centrale nella città, si prestava egregiamente a ospitare la biblioteca. La raccolta libraria del conte Pertusati (risolte le pendenze con il figlio, il conte Luca, in lite con la Congregazione dello Stato per il mancato rispetto delle norme riguardanti i volumi duplicati, che voleva trattenere presso di sé) andò così a unirsi al cospicuo nucleo già appartenente ai Gesuiti. Dodici anni furono necessari alla catalogazione dei fondi, incrementati dall'avocazione al demanio delle raccolte delle altre Case milanesi. Le opere in duplicato vennero assegnate ad altre biblioteche, principalmente a quella del Collegio dei Nobili di Milano, a quella dell'Università di Pavia e a quelle di Cremona e di Lodi. Nel '78 giunse poi a Brera, passato il Gottardo a dorso di mulo, la ricchissima biblioteca del medico e filosofo svizzero Albrecht von Haller.

La Biblioteca di Brera, che aprì al pubblico nel 1786, rispecchiava così la città e le sue tensioni ancora prima di nascere. Figurarsi dopo: questo «dopo» che è anche nostro, in ragione del confronto sempre aperto tra la città e una delle sue istituzioni capitali, come ogni tanto ci ricorda qualche momento particolarmente conflittuale (c'è bisogno di citare le pagine d'apertura della Vita agra di Bianciardi?). E meriterebbe ricordarsi più spesso che dalla fine del Settecento Milano disponeva già di quello che a Parigi, ma molto più tardi, sarebbe stato il Beaubourg. 
Bene: in questo quadro, non stupisce che anche la letteratura, pena un'assoluta perdita di senso e di credibilità, si dovesse associare, come fece, alla nuova sintonia che il potere sembrava ricercare con l'aspirazione alla «pubblica felicità». Per questo a un mio libro su questi temi, quasi trent'anni fa, diedi il titolo (che mi sembra ancora, se non felice, sicuramente didascalico) di Letteratura delle riforme. Il sottotitolo, Da Beccaria a Manzoni, delineava appunto un percorso disposto a farsi carico, come raramente accaduto in precedenza, dei più gravi temi sociali e civili. E rappresentano perfettamente questa sintesi, nella grande poesia di cui quest'età e questa temperie fu capace, le prime grandi Odi pariniane, dalla Educazione per il contino Imbonati all'Innesto del vaiolo, che - niente a che vedere con l'«arcadia della scienza» - accompagnava a mo' di premessa il pionieristico volume di Osservazioni sopra alcuni innesti del medico Giovan Maria Bicetti de' Buttinoni, sino a quella Canzone dedicata all'illustrissimo signor don Pier Antonio Wirtz de Rudenz, ossia Il bisogno, che proprio nell'anno decisivo per la consacrazione internazionale di Dei delitti e delle pene, il 1766, ne riprendeva alcuni punti focali, come la necessità della prevenzione sociale del crimine e la ripulsa delle «atroci pene» comminate dalla recente barbarie legislativa. Con accenti di icastica violenza e con un lessico che non esitava ad aprirsi alle teorie recenti del contrattualismo:

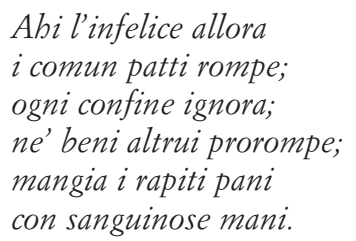

Che è quanto basta a farci considerare, dunque, come non fosse un caso che la pubblicazione del Mattino e del Mezzogiorno cadesse proprio - fra il 1763 e il ' 65 - nello stesso momento di massima apertura dell'«école de Milan» alla lezione delle nuove idee, e appunto in concomitanza al successo di Dei delitti e delle pene e alla pubblicazione del «Caffè» (si ricordi che la raccolta in volume della rivista si chiude proprio con il lungo articolo di Pietro Verri sull'Innesto del vaiuolo). Con i debiti distinguo, naturalmente. Non mancano, nei testi di Verri e di Beccaria, precisi spunti nella direzione dell'egualitarismo e della polemica antinobiliare, ma l'altissima poesia delle prime parti del Giorno (come è noto, il Vespro e la Notte saranno editi, postumi, soltanto nei 
primi anni del nuovo secolo) non vuol certo farsi il loro corrispondente speculare in versi: nella figura del «giovin signore», che «da tutti servito, a nulla serve», non si è anzi stentato a riconoscere, come è noto, qualche traccia della cipria che cospargeva le aristocratiche acconciature dei compagni d'avventura del «Caffè». La formidabile presenza dell'"uomo" Parini nella sua poesia richiede anche, costantemente, un'attenzione al dato "sociale", certo, inteso nel senso più ampio, ma insieme la comprensione della specificità tutta letteraria del suo fronte d'azione. Quella di Parini rimaneva in fondo - come abbiamo ascoltato dalle parole di Pietro Verri quando a proposito della sua formazione richiamava l'epoca trascorsa nell'Accademia dei Trasformati, di cui Parini fu magna pars -, un'altra Milano.

Non andrà però neppure sottovalutata la funzione stessa dell'Accademia, ultimo elemento su cui portiamo l'attenzione: anche per quanto la restituisce alla prospettiva muratoriana da cui abbiamo preso le mosse, nel senso cioè della consapevolezza dell'appartenenza a una tradizione, che inserisce un dato essenziale, di insostituibile specificità, nella ricostruzione che si è tentata. Dietro la riappropriazione del nome di «Trasformati» e dell'antica impresa del platano («ornato di molti frutti, col motto preso da Virgilio Et steriles Platani malos gessere valentes», dal secondo libro delle Georgiche) che furono dell'antica istituzione cui diedero lustro i nomi di Camillo da Rho e del Maioragio (e che nella sostanza raccomandava quasi intera la propria memoria a un volumetto di Sonetti presentati al 1548 all'Infante don Filippo), non si poteva infatti non cogliere la precisa volontà di collocarsi appunto nel solco di una tradizione già illustre, e di portare quindi a confronto con quella la nuova realtà milanese, ora che i tempi sollecitavano a occuparsi «delle scienze e delle belle lettere insieme», come ne scrisse il cronista ufficiale Giorgio Giulini. E fu così decisiva, per il successo che si stava preparando all'istituzione, l'iniziativa di Domenico Balestrieri, promotore nel 1738 di una scherzosa raccolta di Lagrime in morte di un gatto (a stampa nel '41), che sotto il velame dell'ennesimo abuso commesso in nome della voga dilagante delle raccolte poetiche mirava alla loro ridicolizzazione, rendendo evidente il senso antiarcadico di quell'operazione. Ma già nel 1766, a poco più di vent'anni dal ristabilimento, sappiamo che l'Accademia non godeva più, come documentava nel suo Voyage en Italie l'astronomo Joseph-Jérôme de Lalande, «de la même considération qu'autrefois».

Fu proprio una miscellanea in morte di Balestrieri, nel 1780, a riuni- 
re per l'ultima volta alcuni fra i nomi già iscritti nell'albo dell'accademia: con Parini, anche quello dell'antico disertore Pietro Verri, che in onore dell'amico scomparso era tornato a toccare le corde della sua lira, «per lungo disuso inaridite». Anche in partibus infidelium si riconosceva così nella filiazione da Carlo Maria Maggi - che in prima persona viene presentato mentre indirizza, nelle ottave seguenti, il suo discorso a Balestrieri - il senso del percorso compiuto dalle patrie lettere:

\author{
Respira alfin: qui della pace è il regno, \\ qui l'error non compare in faccia al vero, \\ di povertade non v'è il giogo indegno, \\ più il bisogno non v'è tristo e severo; \\ uopo non hai d'affaticar l'ingegno, \\ piegandolo a un servile ministero. \\ Respira alfin la dolce aura di pace, \\ ogni cura è lontana, e là sen giace. \\ [...] \\ Cosi il Maggi dicea là su la soglia \\ dell'Elisio soggiorno fortunato; \\ Entra pur meco, di vederti ha voglia \\ il cantor di Goffredo, il gran Torquato; \\ né temer che il buon Tasso abbia mai doglia \\ se l'hai comicamente trasformato, \\ piaccion l'Armi Pietose in tua favella, \\ che beltà vera in ogni arnese è bella..
}

Versi non eccelsi, ma che ci dicono perfettamente, nell'incrociarsi dei destini e delle scelte, come l'avvenuta saldatura di una poesia che aveva saputo farsi carico delle «materie più utili alla società» con le istanze di rinnovamento maturate nelle biblioteche, negli studi degli storici e nei gabinetti degli scienziati, fra le algide cifre dell'economia e della statistica, fosse di fatto riuscita a convivere con le ragioni, più immediate e battagliere, del gruppo del «Caffè». Per questo credo che anche alla Milano dell'età teresiana possa ben adattarsi l'interpretazione di quell'epoca che ci consegna, in assoluta singolarità, il testo del cosiddetto «Anonimo di Gotha» - una paginetta mai attribuita, di mano non colta, datata a quattro anni dopo la morte di Maria Teresa, il 1784 , e ritrovata un secolo dopo, casualmente, nel campanile della chiesa di Santa Margherita, a Gotha, nella Turingia -, Il secolo felice: 
Siamo vissuti nei giorni più felici del diciottesimo secolo. Imperatori, re e principi scendono affabilmente dai loro temuti altissimi luoghi, disprezzano pompa e splendore, diventano padri, amici e confidenti del loro popolo. La religione fa a pezzi la veste clericale e appare nella sua divina essenza. Il rischiaramento procede con passi da gigante. [...] Le arti e le scienze fioriscono, il nostro sguardo penetra a fondo nel laboratorio della natura. Artigiani e artisti si avvicinano alla loro perfezione, nozioni utili germogliano in tutte le classi sociali. Ecco un federale panorama della nostra epoca. Non siate superbi nel giudicarci, se un giorno starete più in alto e guarderete più lontano di noi. $\mathrm{Ma}$ dal quadro fornitovi riconosciate con quale coraggio e forza noi abbiamo innalzato e consolidato la vostra posizione. Fate lo stesso per i vostri discendenti e siate felici. 